\section{Les cellules du muscle chantent en chcur une berceuse pour cellules souches}

Rana Abou-Khalil, Terry Partridge, Bénédicte Chazaud
R. Abou-Khalil, B. Chazaud:

Inserm U567, Paris, 75014, France;

Institut Cochin, CNRS, UMR8104, Paris, 75014, France ;

Université Paris Descartes, Paris, 75014, France.

Département génétique et développement,

24, rue du Faubourg Saint-Jacques, 75014 Paris, France.

benedicte.chazaud@inserm.fr

T. Partridge : Center for Genetic Medicine,

Children's National Medical Center,

Washington DC 20010, États-Unis.
Cellules satellites, précurseurs myogéniques et cellules de réserve dans le muscle Le muscle strié squelettique adulte peut se régénérer grâce à une population de précurseurs, les cellules satellites. Dans le muscle adulte normal, les cellules satellites sont quiescentes et logées le long des fibres musculaires [1]. Leur capacité à assurer à la fois le renouvellement continu des noyaux des fibres musculaires et la régénération musculaire suggère l'existence de mécanismes qui permettent de maintenir un réservoir de cellules satellites tout au long de la vie [2]. In vivo, la preuve de l'autorenouvellement des cellules satellites a été obtenue après greffe de fibres isolées [3] ou de populations de cellules satellites quiescentes [4]. Des études ex vivo faites sur des fibres isolées qui étaient en phase de prolifération ont montré qu'un petit nombre des cellules précurseurs myogéniques $(\mathrm{mpc}$ ) issues de la division des cellules satellites ne s'engagent pas dans la différenciation terminale mais constituent une réserve des cellules progénitrices sans qu'on puisse, dans ces conditions expérimentales de manipulation de fibres isolées, les nommer cellules satellites [5]. En culture, on observe également cette propriété d'autorenouvellement, puisqu'une sous-population des mpc constitue les «cellules de réserve » $(R C)$ : ces cellules qui ne sont pas engagées dans le cycle cellulaire et sont indifféren-

Le titre de cet article est la traduction du commentaire de S. Duguez et T. Partridge publié dans Cell Stem Cell [12] : Skeletal muscle sings a choral stem cell lullaby. ciées peuvent donner naissance à des cellules différenciées et à de nouvelles $\mathrm{RC}$ dès lors qu'elles sont réensemencées et activées; elles expriment plusieurs des caractéristiques des cellules satellites ${ }^{1}$. Les cellules mpc en phase de prolifération coexpriment les facteurs de transcription Pax7 et MyoD. Puis elles suivent deux destins : la majorité d'entre elles sort du cycle cellulaire et s'engage dans la différenciation myogénique terminale (que caractérisent la répression de Pax7 et la surexpression de MyoD) pour fusionner en myotubes. Une minorité sort du cycle cellulaire pour entrer en quiescence, réprime l'expression de MyoD et surexprime Pax7, arborant une signature caractéristique des cellules satellites quiescentes $\left(\operatorname{Pax} 7^{+} / M_{y o D}{ }^{-}\right)$ [5]. En conséquence, les cellules satellites, ou une sous-population d'entre elles, sont considérées comme les cellules souches du muscle $[3,6,7]$. Cependant, les mécanismes qui contrôlent, dans les précurseurs myogéniques, la sortie du cycle cellulaire et l'entrée en quiescence restent très peu connus.

\section{Le dialogue entre les cellules} satellites et les cellules endothéliales Les cellules satellites sont localisées près des capillaires sanguins et nous avons montré in vitro que les cellules endothéliales $(C \varepsilon)$ et les mpc établissent des interactions paracrines privilégiées, stimulant la myogenèse pour les premières et l'angiogenèse pour les secon-

\footnotetext{
${ }^{1}$ On ne peut toutefois pas les qualifier de cellules satellites, cette appellation reposant sur l'existence d'une localisation anatomique précise le long d'une fibre musculaire.
}

des, notamment via le VEGF (vascular endothelial growth factor) [8]. Un autre couple ligand-récepteur essentiel à la régulation de l'homéostasie vasculaire est celui que forme les angiopoiétines et le récepteur Tie-2. Les angiopoïétines 1-4 (Angl-Ang4) constituent une famille de facteurs de croissance dont le récepteur commun est Tie-2, un récepteur endothélial à activité tyrosine kinase (le rôle de Tie-l est beaucoup moins clair en dépit de son homologie structurale avec Tie-2). Si la liaison de Angl à Tie-2 permet de maintenir l'intégrité vasculaire, Ang2 fonctionne comme un ligand agoniste ou antagoniste de Tie-2 selon le contexte [9]. Le système Angl/Tie-2 est en fait pléïotrope et a été impliqué dans différentes activités biologiques incluant la survie cellulaire, la prolifération, la migration et la quiescence, en particulier celle des cellules souches hématopoïétiques [10].

\section{Rôle du couple Ang1/Tie-2}

dans la régulation du cycle cellulaire des progéniteurs musculaires et la constitution de cellules de réserve Nous avons exploré le rôle du système Angl/Tie-2 dans la régulation du destin des précurseurs myogéniques, in vitro dans un système de culture de cellules humaines, et in vivo dans des modèles murins [11]. L'expression de Angl et de son récepteur Tie-2 est particulièrement forte dans les cellules de réserve. De plus, l'expression de Tie- 2 est 4 fois plus importante dans les cellules en phase GO du cycle cellulaire que dans les cellules en phase G2/M. In vivo, les cellules satellites quiescentes (isolées de muscle 
Figure 1. Schéma des mécanismes autocrines et paracrines induisant la quiescence des cellules précurseurs myogéniques.

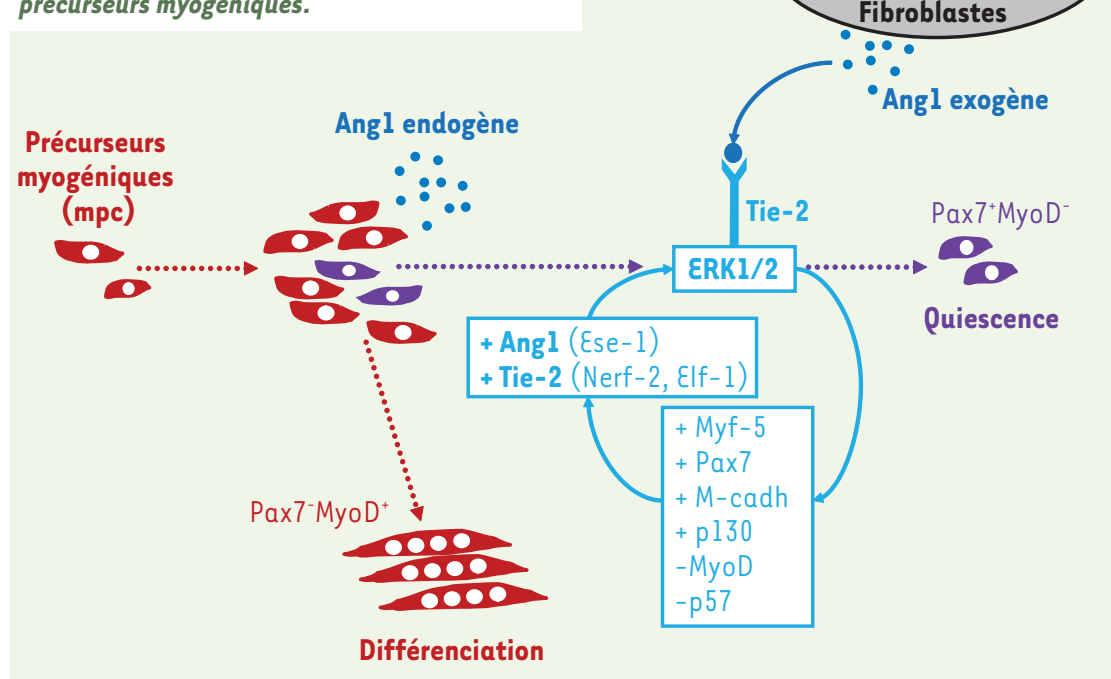

humain par un tri à l'aide de billes magnétisées couplées au marqueur spécifique CD56) expriment Tie-2 et Angl.

L'ajout de Angl à des cultures de mpc inhibe leur croissance ainsi que leur apoptose, via la liaison du ligand à son récepteur Tie-2, et un compétiteur spécifique, Tie-2-Fc, abroge l'effet de Angl sur les cultures. L'ajout de Angl diminue le nombre de mpc en prolifération et celui des cellules différenciées; en outre, il augmente le nombre de cellules en phase GO et donc l'expression des marqueurs associés à la quiescence des cellules myogéniques (Pax7, p130); à l'inverse, les marqueurs associés à la différenciation (MyoD, p57) sont diminués. Ces données montrent que Angl induit la quiescence et privilégie la voie des RC dans les cultures de mpc. Nous avons confirmé ce résultat dans le modèle murin de fibre musculaire isolée, modèle dans lequel on peut suivre le devenir des cellules satellites après leur activation [5]: Angl augmente le nombre de cellules $\mathrm{Pax} 7^{+}$/ MyoD's'engageant dans un processus d'autorenouvellement et diminue celui des cellules Pax $7^{-} /$MyoD $^{+}$en phase de différenciation. Ces effets ont été confirmés par des stratégies d'extinction et de surexpression de Tie-2: l'inhibition de l'expression de Tie-2 dans les mpc par ARN interférence diminue l'expression des marqueurs associés à la quiescence et augmente celle des marqueurs de différenciation, ce qui est corrélé d'un point de vue fonctionnel à une augmentation de la prolifération et de la différenciation. La surexpression de Tie-2 a des effets strictement opposés. Ces données confirment l'influence du couple Angl/Tie-2 dans l'apparition des cellules de réserve et donc dans l'autorenouvellement des cellules satellites.

L'étude des voies de signalisation intracellulaires a montré que la liaison de Angl à Tie-2 active la voie ERKl/2 et régule l'expression de plusieurs gènes associés à la quiescence des cellules satellites, dont Pax7, mais aussi Myf5 et la M-cadhérine.

\section{Angl, un intermédiaire crucial} présent dans l'environnement des précurseurs myogéniques

Les cellules satellites étant situées à proximité des capillaires sanguins, elles sont donc au voisinage des CE et des cellules périvasculaires que sont les cellules musculaires lisses (SMC), les péricytes et les cellules interstitielles de type fibroblastique, et nous avons confirmé la proximité de ces populations par l'analyse in situ de muscle humain adulte. Le milieu conditionné des SMC et des fibroblastes augmente l'expres- sion de Pax7 et, conséquemment, la prolifération des cellules est diminuée. L'addition de Tie-2-Fc dans le milieu abolit cet effet, ce qui indique que l'intermédiaire est l'Angl sécrété par les SMC et les fibroblastes. Cette sécrétion paracrine locale d'Angl s'ajoute donc à la sécrétion autocrine par les cellules de réserve elles-mêmes.

Ensuite, nous avons confirmé cette relation entre degré d'immaturité, quiescence et contrôle par Angl/Tie-2 en analysant les cellules satellites isolées de différentes lignées murines génétiquement modifiées: les souris transgéniques Tie-2-GFP (green fluorescent protein); les souris Pax3/GFP chez lesquelles on peut isoler des cellules satellites quiescentes chez l'adulte et non quiescentes chez le jeune [4]; les souris Myf5-Cre*ROSA-YFP qui permettent d'isoler des populations de cellules satellites Myf5/YFP- et Myf5/YFP+, les premières s'autorenouvelant 4 fois plus que les secondes [6]. L'analyse des populations satellites obtenues après tri cellulaire à partir de ces différentes souches de souris, montre que in vivo, plus les cellules satellites sont quiescentes, plus elles expriment Tie-2. Enfin, des expériences in vivo de gain et perte de fonction ont été réalisées [11]. Dans un muscle en phase de régénération après une lésion, l'expression de Tie-2 a été inhibée par des anticorps bloquants alors que Angl a été surexprimée après électroporation. Les cellules satellites $\mathrm{Pax}^{+}$en phase proliférative ont été analysées en fin de régénération. L'inhibition du système Angl/Tie-2 induit une augmentation du nombre de cellules satellites en cycle alors qu'à l'inverse, la stimulation du système Angl/Tie-2 induit une augmentation du nombre de cellules satellites quiescentes dans le muscle.

\section{Conclusion et modèle}

Nous proposons que la signalisation Angl/Tie 2 est impliquée dans le contrôle du retour à la quiescence d'une souspopulation de précurseurs myogéniques qui assure l'autorenouvellement des 
cellules souches adultes du muscle. Nos données suggèrent une autorégulation de l'homéostasie des cellules myogéniques via une boucle autocrine qui assure la génération de cellules de réserve qui retourneraient en phase de quiescence. La signalisation Angl/Tie-2 permet l'expression d'une série de gènes associés à la quiescence des cellules et réprime ceux qui sont associés à la différenciation (Figure 1). Nos précédents travaux avaient montré que les cellules endothéliales stimulent la croissance des cellules myogéniques et que, inversement, les cellules myogéniques sont proangiogéniques. Le VEGF joue un rôle important dans cette relation bidirectionnelle [8]. On peut proposer que pendant la régénération musculaire, c'est-à-dire lorsque les vaisseaux ne sont pas stabilisés, les cellules endothéliales et myogéniques peuvent interagir entre elles pour promouvoir la myogenèse et l'angiogenèse. Une fois la régénération achevée, l'homéostasie du muscle rétablie et les vaisseaux en voie de stabilisation, la proximité entre les cellules périendothéliales sécrétrices d'Angl et les cellules satellites permet à ces dernières, qui expriment Tie-2, de répondre à la sécrétion de Angl paracrine, ce qui renforce l'état de quiescence. Dans le même temps, Angl stabiliserait les vaisseaux tout en promouvant la quiescence des cellules endothéliales. $\diamond$

How muscle environmental

cells induce stem cells quiescence

\section{CONFLIT D'INTÉRÊTS}

Les auteurs déclarent n'avoir aucun conflit d'intérêts concernant les données publiées dans cet article.

\section{RÉFÉRENCES}

1. Hawke TJ, Garry DJ. Myogenic satellite cells: physiology to molecular biology. J App Physiol 2001 ; $91: 534-51$.

2. Zammit PS, Partridge TA, Yablonka-Reuveni Z. The skeletal muscle satellite cell: the stem cell that came in from the cold. J Histochem Cytochem 2006 ; $54: 1177-91$.

3. Collins CA, Olsen I, Zammit PS, et al. Stem cell function, self-renewal, and behavioral heterogeneity of cells from the adult muscle satellite cell niche. Cell 2005; $122: 289-301$.

4. Montarras D, Morgan J, Collins C, et al. Direct isolation of satellite cells for skeletal muscle regeneration. Science 2005; 309 : 2064-7.

5. Zammit PS, Golding JP, Nagata Y, et al. Muscle satellite cells adopt divergent fates: a mechanism for self-renewal? J Cell Biol 2004 ; 166 : 347-57.

6. Kuang S, Kuroda K, Le Grand F, Rudnicki, MA. Asymmetric self-renewal and commitment of satellite stem cells in muscle. Cell 2007 ; 129 : 999-1010.

7. Perez-Ruiz A, Ono Y, Gnocchi VF, Zammit PS. Beta-catenin promotes self-renewal of skeletal-muscle satellite cells. J Cell Sci 2008; $121: 1373-82$.

8. Christov C, Chretien F, Abou-Khalil R, et al. Muscle satellite cells and endothelial cells: close neighbors and privileged partners. Mol Biol Cell 2007 ; 18 : 1397-409.

9. Shim WS, Ho IA, Wong PE. Angiopoietin: a TIE(d) balance in tumor angiogenesis. Mol Cancer Res 2007 ; 5 : 655-65.

10. Arai F, Hirao A, Ohmura M, et al. Tie-2/angiopoietin-1 signaling regulates hematopoietic stem cell quiescence in the bone marrow niche. Cell 2004 ; 118 : 149-61.

11. Abou-Khalil R, Le Grand F, Pallafacchina G, et al. Autocrine and paracrine Angiopoietin 1/Tie-2 signalling promotes muscle satellite cell self-renewal. Cell Stem Cell 2009; 5: 298-309.

12. Duguez $S$, Partridge T. Skeletal muscle sings a choral stem cell lullaby. Cell Stem Cell $2009 ; 5: 231-2$. 